\title{
Immune Control by Amino Acid Catabolism During Tumourigenesis and Therapy
}

\author{
Henrique Lemos ${ }^{1}$, Lei Huang ${ }^{1}$, George C. Prendergast ${ }^{2}$ and Andrew L. Mellor ${ }^{{ }^{*}}$.
}

*Corresponding author: tel; 01912088503 email; andrew.mellor@ncl.ac.uk

Author affiliations: ${ }^{1}$ Institute of Cellular Medicine, Faculty of Medical Sciences, Framlington Place, Newcastle University, Newcastle-upon-Tyne NE2 4HH. '2Lankenau Institute for Medical Research, 100 East Lancaster Avenue, Wynnewood, PA 19096 USA. Ph: 484.476.8400; Fax: 484.476 .8533 


\begin{abstract}
:
Immune checkpoints arise from physiologic changes during tumourigenesis that re-programme inflammatory, immunologic and metabolic processes in malignant lesions and local lymphoid tissues, which constitute the immunologic tumour microenvironment (TME). Improving clinical responses to immune checkpoint blockade will require deeper understanding of factors that impact local immune balance in the TME. Elevated catabolism of the amino acids tryptophan (Trp) and arginine (Arg) are common TME hallmarks at clinical presentation of cancer. Cells catabolising Trp and Arg suppress effector $T$ cells and stabilize regulatory $T$ cells (Tregs) to suppress immunity in chronic inflammatory diseases of clinical significance, including cancers. Processes that induce Trp and Arg catabolism in the TME remain incompletely defined. Indoleamine 2,3 dioxygenase (IDO) and arginase (ARG1), which catabolise Trp and Arg respectively, respond to inflammatory cues including interferons (IFNs) and TGF $\beta$ cytokines. Dying cells generate inflammatory signals including DNA, which is sensed to stimulate the production of type I IFNs via the Stimulator of Interferon Genes (STING) adaptor. Thus, dying cells help establish local conditions that suppress anti-tumour immunity to promote tumourigenesis. Here we review evidence that Trp and Arg catabolism contributes to inflammatory processes that promote tumourigenesis, impede immune responses to therapy and might promote neurologic comorbidities associated with cancer.
\end{abstract}




\section{[H1] Introduction}

Loss of cell growth control and increased cell survival are necessary but insufficient adaptations that precede malignant tumour formation, as benign pre-malignant lesions develop in tissues of many people who do not get cancer ${ }^{1}$. In most people, inflammation associated with pre-malignant lesions activates local innate immune surveillance mechanisms that prevent benign lesions from transitioning into malignant tumours ${ }^{2}$. In rare cases, cancer develops because local immune regulation becomes dominant, allowing pre-malignant lesions to evade immune surveillance and form malignant tumours. Immune evasion occurs early in tumour development, in part by activation of immune checkpoints creating robust barriers to anti-tumour immunity. How developing tumours evade immune surveillance is poorly understood, as it is difficult to detect pre-malignant lesions and determine their impact on local immune processes as well as draining lymphoid tissues.

At clinical presentation, immune checkpoints are well established and hint at pathways that promote immune evasion (Fig. 1). At early stages of tumour development local immune effector processes that mediate immune surveillance are overcome progressively by tolerogenic (immune regulatory) processes that become dominant in malignant lesions and in tumourdraining lymph nodes (TDLNs), which together constitute the immunologic tumour microenvironment (TME). In essence, the TME is a site of chronic immune activation where immunogenic and tolerogenic processes are activated constitutively, reflecting Dvorak's 1986 comment that malignancies are "wounds that do not heal" 3 . Chronic immune activation provides growth factors that promote tumour growth and angiogenesis and also suppresses anti-tumour immunity. Viewed from this perspective, therapeutic success depends on killing tumour cells, skewing local immune balance in favour of sustained dominance of immunogenic processes that kill tumour cells faster than they can be replaced and preventing re-emergence of dominant tolerogenic processes after therapy ceases. The final point is important because any tumour cells surviving initial therapy would benefit from a resurgent tolerogenic TME that nurtures tumour re-growth and metastasis.

In this review, we focus on increased local catabolism of the essential amino acid tryptophan (Trp), a common local feature that attenuates anti-tumour immunity in primary tumour lesions and TDLNs. Arg catabolism has also been linked to suppression of anti-tumour immunity; however, as less is known about Arg catabolism, and as its role in generating bioactive polyamines that impact cancer was discussed in a recent review, ${ }^{4}$ we will only discuss Arg briefly here. Amino acid catabolism is thought to reinforce immune checkpoints that suppress 
adaptive $\mathrm{T}$ cell immunity. Consistent with this notion, several drugs that act by different mechanisms to inhibit indoleamine 2,3 dioxygenase enzyme activity, which catalyzes the first step of Trp catabolism, have yielded encouraging clinical responses in initial Phase II trials ${ }^{5,6}$. However, the first IDO inhibitor drug to be studied in a Phase III trial did not synergise with immune checkpoint inhibition (PD-1 pathway blockade) to improve clinical outcomes in melanoma ${ }^{7}$, emphasizing the need for more research to understand how amino acid catabolism contributes to immune checkpoints that control immune balance during tumour development and following therapy.

\section{[H1] Amino acids and tumour growth}

As protein building blocks amino acids are essential for tumour growth, and for re-modeling stromal and vascular architecture in the inflamed TME as tumours develop. Hence, it appears counter-intuitive that elevated Trp and Arg catabolism would promote tumour growth. However, immune cells also need amino acids to expand and differentiate into effector cells capable of killing tumour cells. These considerations highlight the need for deeper understanding of how cells sense amino acids and adjust their functions accordingly when access to amino acids is limited. Suppressing immunity by catabolizing Trp and Arg might be more important for tumour development than constraints on tumour growth due to reduced access to these amino acids. Cells could also adapt to compensate for reduced amino acid access, for example by upregulating amino acid transporters and tRNA-charging enzymes. Of note, the Trp-tRNA charging enzyme (WARS) is uniquely induced by type II interferon (IFN $\gamma$ ), suggesting that enhanced access to Trp might help cells survive in inflamed tissues ${ }^{8}$.

[H2] Amino acid catabolism activates cellular stress responses

Cells sense amino acids via two ribosomal kinases, the elF2 $\alpha$ kinase GCN2 and mTOR. When access to amino acids is limiting GCN2 and/or mTOR activate and stimulate radical reprogramming of cell functions, leading to cell cycle arrest and autophagy. GCN2 senses binding of uncharged tRNA to ribosomes and shuts down mRNA translation and promotes cell autophagy, which releases amino acids and makes cells more resilient to nutrient stress ${ }^{9}$. GCN2 is required for optimal growth of sarcoma cells in mice and in culture, suggesting that the integrated stress response (ISR) driven by activated GCN2 promotes sarcoma growth ${ }^{10}$. mTOR is also required for optimal tumour growth in many models, though sensing amino acids is only one aspect of metabolic control by mTOR ${ }^{11}$. Dividing tumour cells must increase amino acid transport to make new proteins and several studies identified links between amino acid transporters, mTOR activity, metabolic re-programming and tumour growth ${ }^{12-14}$. In the mouse 
DBMA/TPA model of inflammatory skin carcinogenesis and a KRAS-induced model of lung tumorigenesis, Ido1 gene expression was required for optimal tumour development ${ }^{15,16}$. These genetic data reinforced pharmacological evidence that 1-methyl-D-tryptophan (D-1MT) could reverse the immune suppressive effects of IDO activity by inhibiting mTOR activation to promote cell autophagy via a GCN2-independent mechanism ${ }^{17,18}$. Tumour cells might compensate for reduced access to amino acids by up-regulating expression of amino acid transporters and by modulating their sensitivity to amino acid sensors that activate GCN2 and mTOR signaling.

Initial indications that Trp catabolism promoted tumour growth emerged from studies in mice treated with IDO inhibitors, which slowed growth of subcutaneous Lewis Lung Carcinoma (LLC) and B16 melanoma tumour grafts and in autochthonous HER2-induced breast tumours ${ }^{19-21}$. In the subcutaneous tumour models, tumour cells did not express IDO but tumour formation induced sustained elevation of IDO activity in dendritic cells (DCs) located in inflamed TDLNs ${ }^{22}$. In the HER2 breast tumour model, IDO inhibitors were most potent when combined with DNAdamaging radio or chemotherapy ${ }^{20}$, prompting significant interest in targeting IDO as novel approaches to boost clinical responses after these treatments.

\section{[H1] Inflammation induces IDO1 and ARG1}

Tumour-associated inflammation stimulates Trp and Arg catabolism, with consequences for tumour development, therapy and comorbidities associated with some cancers (Fig. 2). Increased cell death is a common feature of aseptic inflammation in the TME that generates damage-associated molecular patterns (DAMPs), including DNA. Microbial and mitochondrial DNAs are sensed by Toll-Like receptor-9 (TLR9), which is expressed by specialized antigen presenting cells (APCs) ${ }^{23}$. In contrast, many cells express cytosolic DNA sensors such as the nucleotidyltransferase cyclic GMP-AMP synthase (cGAS), which activate the adaptor Stimulator of Interferon Genes (STING), a potent IFN $\beta$ inducer ${ }^{24}$. In common with many DAMP sensors, DNA sensors like cGAS activate innate immune cells, which express cytokines that can stimulate or suppress immunity. Many innate immune cells produce IFN $\alpha \beta$ and IFN $\gamma$, and IFNs in turn stimulate many downstream genes, including genes encoding enzymes that catabolize Trp (see below) and genes encoding other cytokines that enhance Arg catabolism such as TGF $\beta^{23}$.

[H2] Genes encoding enzymes that catabolise tryptophan

In humans and mice, two unrelated enzymes IDO1 and tryptophan 2,3 dioxygenase (TDO) catalyze the initial step of Trp catabolism to generate a series of downstream catabolites, some 
of which, like kynurenine (Kyn) are secreted. Two isotypic and closely linked genes IDO1 and IDO2 encode IDO apoenzymes, which form holoenzymes with haem (tetrapyrrole) cofactors. IFN $\alpha$, IFN $\beta$ and IFN $\gamma$ are potent IDO1 inducers but regulation of IFN signaling restricts IDO1 expression to particular cell types. IDO2 is largely refractory to IFN signaling but Kyn enhances IDO2 transcription and other evidence points to roles for IDO2 in immune cells ${ }^{25}$. In contrast, TDO gene expression responds to dietary intake in some liver cells and to glucocorticoid stress in other cells ${ }^{26}$. Increased Trp catabolism in cancer patients might have adverse effects on mood and cause depression because Trp is the obligate precursor for synthesis of the neurotransmitter serotonin (also known as 5-hydroxytryptamine, 5HT) and the hormone melatonin, which regulate mood and depressive behaviours. Moreover, some Trp catabolites are neuro-active, including quinolinic (QA), kynurenic (KA) and picolinic acids (PA) ${ }^{27}$. Arg is catabolized by arginase (ARG1) and nitric oxide synthetase (NOS). Nitric oxide is a vasodilator, impacts immune functions and inactivates IDO, as well as other enzymes containing haem cofactors ${ }^{28}$. The ARG1 product L-ornithine can be converted into polyamines, which regulate cell proliferation, and might influence tumour development and tumour immunity ${ }^{4,29}$. IFN $\gamma$ stimulates inducible NOS (iNOS) and T helper 2 (Th2) cytokines (IL-4, IL-13) induce ARG, while IDO1 and ARG1 genes are both responsive to the immune suppressive cytokine transforming growth factor $\beta$ (TGF $\beta)^{30,31}$.

Expression of IDO1 and TDO in tumours are of chief interest for therapeutic targeting, as elevated IDO1 and TDO expression manifests in many cancer types ${ }^{32-35}$. Our unpublished analyses [Lei: OK] of public databases (e.g. kmplot.com \& proteinatlas.org) reveal that relatively high IDO or TDO expression correlate with poor patient survival in some cancers (e.g. lung, acute myeloid leukemia (AML), gastric) but not others (e.g. breast). However, enzyme activity was assessed in few studies, a key point as gene expression might not enhance enzyme activity ${ }^{33,35}$. IDO1 is often overexpressed in tumour cells through genetic and epigenetic mechanisms of pathogenic significance, such as RAS oncogene activation, pro-inflammatory COX2 activation and BIN1 tumour suppressor inactivation ${ }^{36}$. Elevated IDO enzyme activity occurs in many human cancers, including those of the esophagus, stomach, pancreas, colon, lung, breast, ovary, prostate, bladder, skin, and brain ${ }^{37-42}$, though it is not always clear if elevated IDO expression occurs in tumour, immune or stromal cells. IDO1 expression also correlated with poor clinical outcomes after therapy in multiple tumour types ${ }^{39,43,44}$. Though TDO is not expressed in immune cells, TDO elevation in tumour cells, for example human glioblastoma cells, might promote immune suppression in the TME that enhances tumour 
growth ${ }^{45}$. TDO elevation could also contribute to pathogenesis by non-immune mechanisms in tumours such as breast cancer, where IDO1 expression is typically but not always absent ${ }^{46,47}$. Some human tumours express both IDO1 and TDO ${ }^{32}$ and concerns that tumours might exploit TDO as an escape pathway prompted development of drugs that block both enzymes.

\section{[H1] Trp and Arg catabolism affects T cells}

Access to amino acids is necessary for resting (naïve) $T$ cells to proliferate and differentiate into effector cells. After stimulation, $T$ cells rapidly up-regulate an array of amino acid transporters, including SLC7A5. Ablating SLC7A5 blocked metabolic re-programming via activation of mTOR, and expression of the transcription factor MYC to prevent $T$ cell proliferation ${ }^{48}$, revealing a critical need for enhanced amino acid access to sustain $\mathrm{T}$ cell responses after priming. However, $T$ cells have differential requirements for specific amino acids, as depletion of Trp or Arg activated GCN2 to block entry into S-phase following T cell stimulation, while depletion of both isoleucine (Ile) and leucine (Leu) led to cell cycle entry (S/G1 transition), though $\mathrm{T}$ cells stopped dividing and died soon afterwards ${ }^{49-51}$. These distinctive responses suggest that $T$ cell access to sufficient Trp and Arg are key signals required for cell cycle entry, while restricted access to other amino acids does not block cell cycle entry but causes catastrophic nutrient 'starvation' soon after cell cycle entry. It may not be a coincidence that elevated Trp and Arg catabolism are common TME hallmarks. These adaptations could represent an evolved counter-regulatory 'healing' response to restrain immune-mediated injury in inflamed tissues where immune-activation thresholds are reduced. Malignant lesions might hijack tissue healing responses to suppress anti-tumour immunity and create robust immune checkpoints.

Previous reviews describe how elevated Trp and Arg catabolism inhibits adaptive immunity by suppressing effector $\mathrm{T}$ cells and potentiating FOXP3-lineage regulatory CD4+ $\mathrm{T}$ cell (Treg) functions ${ }^{33,35}$. In brief, tumour or stromal cells in malignant lesions catabolizing Trp and/or Arg suppress CD8+ effector T cells and stabilize Treg cells to protect tumour cells. Similarly, in TDLNs, APCs catabolizing Trp and Arg suppress expansion of conventional CD4+ helper T cells and tumour-specific effector CD8+ T cells and stimulate and stabilize Tregs (Fig. 1). The combined actions of Trp and Arg depletion and Trp catabolite production at these discrete TME sites create robust barriers to anti-tumour immunity that complement immune checkpoints mediated by CTLA4 and PD-1-PD-L1 pathways in T cells. For example, the PD-1 and IDO pathways synergized to stabilize Tregs in the B16 mouse melanoma model ${ }^{52}$. While PD-1 signaling maintained PTEN activity to prevent PI3K activation, IDO activity activated GCN2 to 
block mTOR complex 2 (mTORC2) activation in Tregs. These metabolic pathways synergized to suppress AKT signaling, thereby preventing de-stabilization of Treg regulatory functions in the inflamed TME. The distinctive roles of PTEN as a tumour suppressor in T-ALL and a Treg stabilizer in the B16 melanoma model emphasize that a particular pathway can be active in multiple TME cell types and have differential effects on tumour growth or responses to therapy in specific models.

Tregs can undergo re-programming to provide helper or effector immune functions if they receive appropriate inflammatory signals. Physiologic factors driving Tregs to adopt diametric functions remain poorly defined but are important, as they will provide novel insights on how to improve clinical responses in cancer patients by skewing the local immune balance in the TME in favour of immunogenic processes that target tumour cells. Since local Trp and Arg catabolism activates and stabilizes Treg functions, it follows that inhibiting these metabolic pathways might de-stabilize Tregs to weaken a formidable barrier to anti-tumour immunity in the TME. For example, Tregs unexpectedly provided 'helper' functions to prime effective T cell responses to a tumour vaccine in naïve mice but 'Treg help' was suppressed via an IDO-dependent mechanism in mice with established B16 melanomas ${ }^{53}$. Stability might also differ amongst Treg subsets. In mice harbouring B16 melanomas, TDLN DCs expressing IDO activated a PD- $1^{+}$Treg subset via a GCN2-dependent mechanism to stabilize the transcription factor EOS (also known as IKZF4), an obligate FOXP3 co-repressor, and prevent Treg re-programming ${ }^{54,55}$. Thus, the sensitivity of some Tregs to Trp and Arg catabolism reveals potential targets to promote Treg reprogramming, and to weaken immune checkpoints in cancer patients.

\section{[H1] Catabolism of Trp and Arg in the TME}

T cells and Tregs respond to Trp and Arg catabolism; thus, degradation of these amino acids by tumour or accessory cells expressing IDO, TDO and/or ARG regulate T cell immunity in the inflamed TME. This scenario, in which interactions between distinct cell types regulate immune balance in the TME, mirrors how accessory cells expressing PD-1 ligands regulate $T$ cell immunity. Some, but not all, tumour cells have elevated IDO, TDO, ARG, and iNOS enzyme activity, and in some cases resident stromal cells and/or infiltrating immune cells express these enzymes to promote Trp and Arg catabolism in the TME.

\section{[H2] Monocytic cells that catabolize Trp and Arg}

Monocyte-derived cells such as DCs and macrophages are innate immune cells with pivotal roles in promoting and regulating adaptive immunity. Due to sustained inflammation in the TME, 
monocyte-derived cells found in the TME can exhibit distinctive phenotypes that differ from circulating peripheral blood monocytes (PBMCs) and APCs found in homeostatic tissues (Fig 1). For example, in mice with subcutabeous B16 melanoma or LLC tumour grafts, a discrete DC subset with potent $T$ cell regulatory attributes (DCregs) exhibited IDO1 activity in TDLNs and displayed features of both $B$ cells and plasmacytoid DCs ${ }^{22,56}$. Some DCs generated from human PBMCs can also express IDO1 and regulate T cell immunity in response to inflammatory signals ${ }^{57,58}$. Moreover, elevated numbers of IDO1-expressing DCs were observed in sentinel lymph nodes from some melanoma patients ${ }^{59}$ consistent with the notion that TDLN DCs expressing IDO1 regulate T cell immunity in some cancer patients.

Myeloid-derived suppressor cells (MDSCs) with macrophage and granulocyte-neutrophil attributes infiltrate many primary tumour lesions in humans and mice. MDSCs often express ARG, reflecting their potential origin as alternatively activated M2 macrophages ${ }^{60,61}$. Some MDSCs also exhibit other immune regulatory hallmarks, including IL-10, TGF $\beta$ and IDO ${ }^{16}$. In mice and humans with melanoma, IDO activity in tumour cells promoted MDSC recruitment and activation via a Treg-dependent mechanism in the TME ${ }^{16,62}$. Accordingly, IDO inhibition reduced MDSC and Treg numbers and diminished their regulatory functions. Increased numbers of circulating MDSCs expressing ARG1 were also observed in glioblastoma patients 63 . Fibrocytes that expanded and circulated amongst PBMCs in cancer patients also suppressed mitogen-activated T cell proliferation ex vivo, primarily via IDO, unlike circulating fibrocytes from healthy donors that potentiated $\mathrm{T}$ cell proliferation ${ }^{64}$. Thus, DCs, MDSCs, and circulating fibrocytes catabolizing Trp and Arg mediate tolerogenic processes in the TME. Collectively, these inflammatory cells contribute to barriers to natural and therapy-induced anti-tumour immunity, even if tumour cells themselves do not catabolize Trp or Arg.

\section{[H1] DNA sensing and Trp catabolism}

[H2] DNA from dying cells is a potent immune tolerogen

Elevated Trp and Arg catabolism are common features of the TME in humans and mice but mechanisms linking tumour growth to increased Trp and Arg catabolism are poorly understood. Inflammatory pathways are strong candidates, as inflammatory cytokines stimulate IDO1, ARG and iNOS expression (Fig. 2). Moreover, some DCs in mice and humans express IDO in response to IFNs and suppress $T$ cell responses ${ }^{57,58,65}$. Further hints emerged from studies on immune responses to DNA nanoparticles and apoptotic (dying) cells in mice 66-70. These treatments induced robust IDO1-dependent tolerogenic responses that alleviated autoimmune 
diseases and, in the case of apoptotic cells, protected skin allografts. Notably, in each case tolerogenic responses to DNA nanoparticles and apoptotic cells were STING dependent.

[H2] DNA from dying tumour cells is sensed to activate STING and induce Trp catabolism

Subcutaneous LLC tumour cell engraftment led to rapid and sustained elevation of IDO activity in tumour lesions and TDLN DCs via STING-dependent induction of IFN $\alpha \beta$ signaling but not IFN $\gamma$ signaling ${ }^{22}$. Ablating cGAS attenuated IDO induction in some but not all mice, suggesting that other cytosolic DNA sensors that activate STING might compensate for loss of cGAS functions. Consistent with these findings, STING was required for optimal LLC tumour growth in dermal and lung sites, phenocopying requirements for IDO1 to promote optimal LLC growth. STING was also required to induce TDLN IDO activity during EL4 thymoma growth in mice, though STING ablation did not reduce IDO activity in EL4 lesions because tumour cells expressed IDO constitutively. B16 melanomas did not induce IDO activity in TDLNs ${ }^{22}$; a surprising outcome in light of previous reports identifying DCs expressing IDO in TDLNs from mice bearing B16 tumours ${ }^{56}$. However, in earlier studies IDO activity in TDLNs was not assessed directly and mice harboured cohorts of T cells specific for ovalbumin (OVA) expressed by B16 tumour cells. The presence of tumour-specific T cells can provide additional signals (e.g. IFN $\gamma$, CTLA4, PD-1) that induce and sustain IDO activity in DCs ${ }^{71,72}$. Thus, DNA sensors induced IDO in some but not all mouse tumour models. Impaired STING signaling is common in many human cancers and cell lines and defective STING signaling might attenuate immune surveillance to promote tumourigenesis ${ }^{73}$. However, this study did not address if DNA sensors induced IDO or if impaired STING signaling correlated with reduced IDO activity.

[H2] Elevated tumour immunogenicity attenuates tolerogenic responses to DNA

It is unclear why distinct requirements for DNA sensing to induce IDO manifest in different tumour models. One clue emerged from use of LLC tumour cells with elevated immunogenicity due to induced expression of neo-antigens. While parental LLC cells induced IDO to promote tumour growth, LLC cells expressing defined neo-antigens did not induce IDO in TDLNs, and STING signaling either had no effect on tumour growth or attenuated growth of tumours expressing neo-antigens ${ }^{22}$. These outcomes suggest that increased potency of $T$ cell responses to tumour antigens attenuated tolerogenic responses to DNA. As IDO was induced one day after tumour cell engraftment it is unclear how $T$ cell responses could influence this rapid innate immune response to dying tumour cells. This point notwithstanding, elevated tumour immunogenicity clearly skews STING-dependent immune responses to DNA towards inhibition of tumour growth. 
[H2] Cell death influences immune balance in the TME

Other potential factors that could influence immune responses to DNA during tumourigenesis include differential rates and modes of tumour cell death, DNA release, uptake or sensing of DNA by cells in the TME, or redundant requirements for IDO to promote tumour outgrowth. Studies on DNA sensing by mouse bone-marrow derived macrophages (BMDMs) underscore the ability of DNA to drive immune responses in opposing directions. While phagocytic BMDMs that ingested dying tumour cells sensed DNA to activate STING and trigger production of proinflammatory cytokines ${ }^{74}$, BMDMs that ingested apoptotic thymocytes sensed DNA via TLR9, a microbial and mitochondrial DNA sensor, to induce potent tolerogenic responses ${ }^{75}$. Such diametric immune responses to DNA emphasize the need to better understand how distinct cell types in the TME sense DNA to enhance Trp catabolism and to optimise the use of IDO inhibitors to improve clinical responses to DNA-damaging chemotherapies or radiotherapies. Similar considerations may apply to other DAMPs that promote innate immunity. In particular, DAMPs that stimulate IFN $\alpha \beta$ production may induce IDO1 activity downstream, though other DAMPs that induce IFN $\gamma$, IL-10 or TGF $\beta$ production could also enhance Trp and/or Arg catabolism ${ }^{30,76}$. Collectively, these observations underscore the critical role of DNA and other DAMP sensors that activate inflammatory signaling pathways and influence immune balance in the developing TME. Moreover, recognition that DNA could promote tolerogenic or immunogenic responses in the TME has implications for cancer therapies using STING agonists.

\section{[H1] Roles of Trp catabolites}

Some Trp catabolites made downstream of IDO suppress immunity and other Trp catabolites are neurotoxic (Fig. 3). Thus, elevated Trp catabolism could not only attenuate anti-tumour immunity but also might cause undesirable neurologic changes during cancer development or promote neurotoxicities in response to cancer treatment.

\section{[H2] T cell suppression by Trp catabolites}

Kyn, which is exported by many cells expressing IDO, and its downstream catabolite KA are natural aryl hydrocarbon receptor (AHR) agonists, though KA is more effective than Kyn in activating $A H R{ }^{77}$. AHR signaling regulates immunity and induces some DCs to express IDO ${ }^{77-}$ 81. Thus, Kyn and KA might amplify IDO activity via AHR signaling and promote tolerogenic processes in the TME, a paradigm supported by a study on human cancers expressing IDO ${ }^{82}$. AHR signaling has multiple links with cancer, including regulation of oncogene expression, 
angiogenesis, cell survival, as well as effects on immune cell functions ${ }^{83}$. One study showed that the IDO pathway inhibitor D-1MT stimulated immune responses via an AHR-dependent mechanism in mesenchymal stem cells (MSCs) ${ }^{84}$, suggesting that the anti-tumour effects of D1MT may in part depend on AHR activation in the TME, as well as IDO pathway modulation. The interface between Trp catabolism and AHR signaling provides numerous opportunities to modify the TME for clinical benefit, though the central role of AHR signaling in toxic responses to xenobiotic compounds such as dioxin, a synthetic AHR agonist, may be a limiting factor ${ }^{85}$. Downstream of Kyn, 3-hydroxykynurenine (3HK) and 3-hydroxyanthranilic acid (HAA) are also immune suppressive. HAA inhibited pyruvate dehydrogenase kinase (PDK1) and NF-кB activation to block $T$ cell proliferation, promote $T$ cell apoptosis and activate Tregs, though how $T$ cells and Tregs sensed HAA is unclear ${ }^{86,87}$. These studies were not performed in cancer models and it is not known if $3 \mathrm{HK}$ and HAA condition the TME to promote tumour growth and if so, via which mechanisms. A recent study revealed a metabolic link between Trp catabolism and tetrahydrobiopterin (BH4) synthesis in T cells, as the Trp catabolite xanthurenic acid (XA) inhibited $\mathrm{BH} 4$ synthesis required to generate effector $\mathrm{T}$ cells ${ }^{88}$. $\mathrm{BH} 4$ supplementation overcame XA-mediated suppression to boost anti-tumour immunity in mice, suggesting that IDO activity in the TME might block BH4 synthesis in T cells to attenuate anti-tumour immunity.

The previous points raise a key issue, as TME cells express enzymes that further degrade specific Trp catabolites, making it difficult to ascertain which Trp catabolites mediate specific biological effects that impact tumour development, therapy or associated comorbidities. For example IFN $\gamma$ induced human glioma cells to up-regulate IDO1, IDO2 and other genes encoding enzymes in the pathway (KMO, KYNU) but down-regulated others (ACMSD, KATII) ${ }^{89}$. In this study, Kyn:Trp ratios were elevated in serum samples from glioma patients (consistent with elevated IDO activity) but KA, QA and PA levels were all reduced, relative to healthy individuals. These observations implicate Trp catabolism in glioma pathogenesis but also reveal the difficulty of correlating data on gene expression in tumour cell lines with metabolic effects caused by Trp catabolism in cancer patients. Another study in glioma patients showed that QA from brain microglial cells was converted via QPRT expressed in glioma cells into the oxidized form of nicotinamide adenine dinucleotide $\left(\mathrm{NAD}^{+}\right)$, which inhibits apoptosis and increases resistance to oxidative stress and might contribute to glioma survival via these processes ${ }^{90}$. Moreover, chemotherapy and radiotherapy enhanced QPRT expression by glioma cells, which might reinforce glioma cell protection in response to these therapies. Meta-analyses of public databases using an online tool (http://kmplot.com/analysis/index) of genes encoding specific 
enzymes in the Trp catabolism pathway reveals several positive and negative correlations with cancer patient survival, though no uniform picture emerges that applies to multiple cancers. Clearly, elevated Trp catabolism impacts tumour growth, anti-tumour immunity and therapeutic responses in complex ways and improved methods to assess metabolic flux downstream of IDO are needed to understand how Trp catabolism mediates immune suppression and other processes relevant to cancer. Delivering recombinant enzymes that function downstream of IDO to reduce levels of immune suppressive Trp catabolites in the TME is a potential strategy to improve anti-tumour immunity. For example, administering recombinant, pegylated kynureninase (KYNU) to reduce Kyn levels in the TM attenuated immune suppression and promoted more effective tumour control in several mouse models when combined with immune checkpoint inhibitors or a cancer vaccine ${ }^{91}$. Lower Kyn levels may be beneficial because Kyn (and/or downstream catabolites) mediate immune suppression and neuro-toxicity, though it may prove more challenging to reduce Kyn levels to levels that promote clinical responses in patients.

\section{[H2] Elevated Trp catabolism and cancer comorbidities}

As in other chronic inflammatory syndromes, many cancer patients suffer chronic pain, depression, behavioural disorders and fatigue, and some cancer treatments exacerbate these comorbidities ${ }^{92}$. Elevated Trp catabolism in the TME might contribute to these neurologic comorbidities, as some Trp catabolites are neuroactive (Fig. 3). Targeting specific enzymes might help alleviate comorbidities associated with some cancers and cancer treatments such as chronic pain and depression. Trp is the obligate substrate for $5 \mathrm{HT}$ synthesis and $5 \mathrm{HT}$ is an IDO substrate. Hence, sustained Trp catabolism reduces $5 \mathrm{HT}$ levels by removing Trp and degrading $5 \mathrm{HT}$ with consequent negative impacts on mood and behavior ${ }^{93}$. Moreover, targeting Trp catabolism to treat neurologic dysfunction in cancer patients might also help treat tumours, as many cancer cells and cell lines express $5 \mathrm{HT}$ receptors and $5 \mathrm{HT}$ signaling impacts tumour growth, metastasis and angiogenesis ${ }^{94,95}$.

Elevated Trp catabolism during chronic inflammation in cancer and other conditions may cause neurologic dysfunction due to sustained production of neuroactive Trp catabolites ${ }^{96,97 .}$ $\mathrm{QA}$ and KA potentiate and antagonize, respectively, N-methyl-D-aspartate (NMDA) receptor signaling in neurons. In mice, genetic ablation of IDO1 prevented increase in mechanical nociception caused by chronic inflammation in models of LPS-induced arthritis and virus infections, while Kyn enhanced mechanical nociception in mice lacking IDO1 genes ${ }^{96,97}$. Thus, elevated Trp catabolism in the TME might impact neurologic functions adversely before and 
after clinical presentation of cancer, and therapy could alleviate or potentiate these effects ${ }^{98}$. Strategies to manipulate Trp catabolites, especially in the central nervous system, might reduce these comorbidities in cancer patients. However, fatigue and pain were common adverse effects observed in a phase I trial with the IDO inhibitor Epacadostat, though it is unclear how effective this drug was in reducing Kyn levels, as it has a relatively short half life $(2-4 \mathrm{hrs}){ }^{99}$.

\section{[H1] Trp and Arg catabolism in therapy.}

The IDO pathway is an attractive target for intervention, as it affects multiple downstream pathways controlling cell growth, function and viability (Fig. 2). Recent reviews provide detailed discussion of IDO inhibitors in clinical development ${ }^{36,100}$. Here we offer a brief summary of efforts to target IDO and TDO pathways with small molecule inhibitors focusing on the compounds most studied in the clinic. To aid discussion, Table 1 lists all compounds currently under investigation as potential IDO or IDO pathway inhibitor drugs to enhance immunologic responses to cancer.

\section{[H2] STING agonists and Trp catabolism in cancer therapy}

High IFN $\alpha$ and IFN $\beta$ expression in tumour lesions correlates with spontaneous T cell priming by developing tumours ${ }^{101}$. In immunogenic tumour models such as B16 melanoma expressing a neo-antigen (B16.SIY) this correlation occurs because DNA sensors activate STINGdependent IFN $\alpha$ and IFN $\beta$ signaling to enhance antigen cross-presentation by $\mathrm{CD}^{+} \mathrm{DCs}$, which primes robust anti-tumour immunity mediated by $\mathrm{CD}^{+} \mathrm{T}$ cells ${ }^{102,103}$. These observations led to studies using synthetic cyclic dinucleotides (CDNs) that bind and activate STING as anti-cancer therapeutics. Intra-tumoural injection of CDNs induced tumour regression and systemic antitumour immunity in mouse models of melanoma, colon and breast cancer ${ }^{104,105}$. However, half of the mice failed to respond to CDN treatment and eventually succumbed to tumour growth. In addition, therapeutic responses were only partially dependent on IFN $\alpha \beta$ signaling suggesting that other pro-inflammatory pathways might also promote immunogenic responses to CDNs. Though the anti-tumour effects of CDNs were not influenced by tumour immunogenicity, the requirement for STING to retard tumour growth manifested only if high numbers of B16.SIY cells

were implanted ${ }^{22,102,104}$. This suggests that tumour burden and immunogenicity might impact how STING signaling influences immune balance in the TME. Studies in the LLC model support this notion, as parental LLC tumours grew slower and LLC cells expressing neo-antigens grew faster in STING-deficient mice ${ }^{22}$. Thus, CDN treatments might control highly immunogenic ("hot") tumours more effectively because immune checkpoints that regulate anti-tumour immunity are less potent in these settings. How CDNs were administered to mice was also 
critical, as systemic CDN administration induced IDO activity in many mouse tissues; indeed, systemic CDN administration is an effective treatment to alleviate autoimmune arthritis and encephalitis in mice via IDO ${ }^{70,106}$. These issues are clinically important because effective cancer immunotherapy must shift immune balance in the TME in favour of sustained dominance of immunogenic processes by disrupting established tolerogenic processes and - equally important - preventing subsequent re-emergence of tolerogenic processes after therapy (Fig. $1)$.

\section{[H2] Immune balance and adverse responses to therapy}

The paradigm that effective cancer immunotherapy must reorient the immune balance in the TME raises several concerns. First, immunotherapy might shift immune balance too far, eliciting adverse toxicities due to enhanced immunity such as autoimmunity, a toxicity that manifests in some patients after immune checkpoint therapy ${ }^{107,108}$. Exacerbation of pre-existing autoimmune diseases after checkpoint immune therapy has also been reported ${ }^{109,110}$. Second, chemotherapy, radiotherapy and STING agonist treatments might stimulate tolerogenic responses that attenuate desired immunogenic responses. Notably, the efficacy of cytotoxic drugs is enhanced by IDO enzyme blockade in mice ${ }^{20,21,111}$, perhaps via STING agonism ${ }^{105}$. Poor clinical responses to some TLR ligands and tumour vaccines might arise in part because increased IDO activity dampens the innate immune adjuvant effects of these treatments ${ }^{112,113}$. Similarly, IFNs might have limited efficacy as cancer therapies and in some cases enhanced tumour aggression, promoted metastasis and induced significant adverse toxicities, perhaps in part because they induced IDO, which attenuated clinical benefit ${ }^{114-116}$. Lastly, neurologic comorbidities associated with some cancers (e.g. pain, altered behaviour, fatigue) and many cancer therapies (e.g. neuropathy, chemobrain), might be linked mechanistically to elevated Trp catabolism, which can generate neurotoxic catabolites (Fig. 3). These considerations suggest that combining STING agonists with IDO inhibitors might be more effective and less toxic than monotherapies using one drug type. Moreover, this drug combination might be a more potent immunometabolic adjuvant to enhance anti-tumour responses than traditional cytotoxic therapies or immunotherapy (e.g. PD-1 or PD-L1 checkpoint inhibitors), even when these latter treatments are combined with IDO inhibitors.

[H2] Cancer therapies targeting Trp catabolism

The two most advanced drugs in development are indoximod and epacadostat (also known as D-1MT and INCB024360, respectively). Preliminary reports from meeting abstracts relating to other drugs being tested in ongoing clinical trials, include the IDO1 and TDO2 inhibitor 
navoximod (NLG919), which is 10-fold more selective as an IDO1 than TDO inhibitor ${ }^{117,118}$, the selective IDO1 inhibitor BMS-986205 ${ }^{119}$, the IDO1 and TDO2 inhibitor PF-06840003 ${ }^{120}$, the IDO1 inhibitor KHK2455 ${ }^{121}$, and the D-1MT pro-drug NLG-802 ${ }^{122}$, the IDO1 inhibitor LY3381916, and the IDO1 and TDO2 inhibitor SHR9146. As a class, these drugs exhibit little efficacy as single agents and toxicity is limited and manageable. Combination drug trial designs are being evaluated given the strong rationale for using IDO inhibitors as immunometabolic adjuvants to enhance the efficacy of cytotoxic chemotherapy, radiotherapy and immunotherapies.

To date, clinical evaluation of IDO inhibitors for cancer treatment has been driven largely by intense interest in identifying agents that improve the efficacy of immune checkpoint therapies, in particular anti-PD1 therapy. However, potent effects in preclinical tumour models were observed when IDO inhibitors were combined with 'immunogenic' cytotoxic chemotherapy ${ }^{20}$. The rationale for combination immunotherapy is based on the potential for multiple immune checkpoints to be active at clinical presentation, and/or to suppress compensatory responses to monotherapies targeting a single checkpoint or conventional cytotoxic therapies ${ }^{123,124}$. In addition, evidence from preclinical models shows that IDO inhibitors can enhance immune checkpoint therapy ${ }^{125-127}$. Encouraging but preliminary Phase 1/2 data have emerged from studies combining PD1 or PDL1 pathway-inhibitors with: D-1MT for advanced melanoma ${ }^{128-130}$; epacadostat for urothelial carcinoma ${ }^{131}$, renal cell carcinoma ${ }^{132}$, non-small cell lung cancer (NSCLC) ${ }^{133}$, squamous cell carcinoma of the head and neck ${ }^{134}$; BMS-986205 for various advanced cancers 119; and navoximod for advanced solid tumours (NCT02471846) ${ }^{135 .}$ However, data from the first Phase 3 trial (ECHO-301) reported for anti-PD1 and epacadostat in metastatic melanoma revealed that epacadostat did not enhance therapeutic responses to PD-1 blockade ${ }^{136}$. Notwithstanding criticism of this Phase 3 trial ${ }^{7}$ and promising outcomes in early phase trials ${ }^{6}$, the disappointing outcome emphasizes the need for more biomarker analyses and improved mechanistic understanding of how $\mathrm{T}$ cell and metabolic immune checkpoints interact and compensate for each other during therapy.

\section{[H2] Cytotoxic chemotherapies and targeting Trp catabolism}

Many cytotoxic chemotherapies are 'immunogenic' and can enhance immune responses ${ }^{137}$. As noted above, IDO inhibitors synergize powerfully with 'immunogenic' chemotherapy and enhance the efficacy of radiotherapy ${ }^{20,21,138}$. Clinical regimens of immunochemotherapy or immunoradiotherapy that include IDO inhibitors are appealing in principle, to take advantage of synchronous tumour cell death and tumour-related antigen release ${ }^{139}$ that may enhance 
antigen cross-presentation by APCs to stimulate effector T cells in the TME. In a Phase 1 study in metastatic breast cancer patients, the combination of D-1MT and docetaxel was well tolerated with no additive toxicity and an objective response rate of $18 \%$ (4/22 with partial responses) and an additional $45 \%(10 / 22)$ achieving at least transient stable disease ${ }^{140}$; however, results from a follow-on randomized, placebo-controlled phase 2 trial are not yet available. Other early-phase combination drug trials are studying D-1MT in combination with: gemcitabine and nab-paclitaxel for metastatic pancreatic cancer 141,142; temozolomide with or without bevacizumab for temozolomide-refractory glioblastoma ${ }^{130}$; and cytarabine and idarubicin for newly diagnosed $\mathrm{AML}^{143}$. Additionally, a dedicated Phase 1 paediatric trial combining D-1MT with temozolomide for recurrent brain cancer or newly diagnosed diffuse intrinsic pontine glioma patients is ongoing 144,145 . To date, all these combinations have been well tolerated with little indication of any overlapping toxicity with chemotherapy, though it is important to emphasize the preliminary nature of these early reports.

\section{[H2] Radiation therapy and targeting Trp catabolism}

Like many chemotherapies, radiation treatments induce a highly inflammatory milieu in targeted tissues in preclinical studies ${ }^{146}$, including expression of IFNs and other mediators that induce IDO. If potent cytotoxic and immunogenic effects of radiation are combined with IDO inhibitor therapy to enlist targeted immune responses to residual tumour cells, it may be possible to craft less toxic radiation strategies that retain effectiveness with reduced off-target radiation exposure ${ }^{144}$. A recent preclinical study encourages immunoradiotherapy strategies incorporating anti-PD-1 as well as IDO inhibition ${ }^{147}$. Another potential benefit of combining immunotherapy with radiation is the possibility of achieving abscopal responses, where local radiation delivered to one tumour may lead to regression in a distant tumour outside the radiation field ${ }^{148-151}$. Abscopal responses to radiotherapy may be immune-mediated and may reflect high-grade immune priming. To date, few clinical studies have combined IDO-inhibitor therapy (D-1MT) with radiosurgery for adult refractory glioblastoma ${ }^{130}$ and pediatric recurrent brain cancers or newly diagnosed pontine glioma ${ }^{144,145}$. Preliminary results indicate that this combination was well tolerated when followed by cyclic immuno-chemotherapy using D-1MT plus temozolomide. Using IDO inhibitors as immunometabolic adjuvants to radiological and cytotoxic treatment modalities may enhance immune responsiveness with little added toxicity.

[H2] Trp catabolism and cancer biomarkers

Recent clinical findings suggest that biomarker analyses will be critical to determine how IDO inhibitors can be used most effectively. Excitement in the field of cancer immunotherapy has encouraged aggressive entry to large (Phase 3 ) trials that, in retrospect appears premature ${ }^{7}$. 
For example, tumour lesions often generate nitric oxide, (possibly due to iNOS activity), which inhibits IDO activity ${ }^{28}$, emphasizing the critical importance of measuring IDO activity. However, few studies have evaluated IDO activity before and after therapy but instead rely on assessing IDO gene or protein expression. Systemic IDO activity was evaluated by assessing levels of Trp and Kyn in serum samples from patients with NSCLC before, during and after patients received radiotherapy ${ }^{152}$. While many $(65 \%)$ patients with low serum IDO activity before and after radiotherapy were alive 10 years after radiotherapy, all NSCLC patients with relatively high serum IDO activity succumbed within 3 years. Furthermore, IDO activity was reduced during radiotherapy in most NSCLC patients but IDO activity levels increased after radiotherapy in some patients. These findings reinforce the notion that IDO activity contributes to immune checkpoints, show that radiotherapy disrupts this barrier to clinical responses (albeit only transiently in some cases), and suggest that serum Kyn levels may be an informative biomarker of clinical responsiveness to radiotherapy in some cancer patients.

\section{[H1] Conclusions and Future Directions}

Extensive evidence supports the notion that elevated Trp and Arg catabolism contributes to immune regulation that promotes tumour growth and therapy resistance. Targeting these biochemical pathways in cancer patients offers promise and many drugs are under development with this goal in mind. However, setbacks in a recent clinical trial reveal the need for more research to better understand how changes in amino acid transporters, sensors and catabolism impact immune responses in the TME. Biochemical changes associated with increased Trp and Arg catabolism have many effects on the function and viability of tumour, stromal and immune cells in the TME. Other outstanding questions are how Trp and Arg catabolism contributes to other immune regulatory mechanisms in the TME and how immune checkpoint blockade impacts Trp and Arg catabolism. There are also clear unmet needs to identify biomarkers of the potency of amino acid catabolism in cancer patients as ways to identify patients likely to benefit from treatments targeting amino acid catabolism. In this arena, biomarkers to inform patient recruitment to trials of IDO inhibitors are needed, as well as to inform how best to use DNA damaging therapy plus PD1 inhibitors (or other immunotherapy), as an immunometabolic adjuvant. Given the current focus on enhancing anti-tumour immunity, there is enormous interest in gaining more understanding of how chronic inflammation associated with tumourigenesis stimulates local Trp and Arg catabolism that regulates immune responses. Furthermore, it will be important to identify innate and adaptive immune biomarkers for the stimulation of pathogenic amino acid Trp and Arg catabolism during tumourigenesis as potential 
clues to the underlying fundamental processes of immune escape that promote cancer development or relapse after therapy.

Acknowledgements: Research in the ALM and LH laboratory is supported by NIH (AI103347), Cancer Research UK and the Faculty of Medical Sciences at Newcastle University. Research in the GCP laboratory is supported by NIH (CA191191), The W.W. Smith Trust, The Lankenau Medical Center Foundation and Main Line Health. GCP is The Havens Chair in Biomedical Research at the Lankenau Institute for Medical Research.

\section{Author contributions}

All authors researched data for the article, substantially contributed to the discussion of content, and wrote, reviewed and edited the manuscript.

Conflict disclosures: ALM and GCP receive remuneration as scientific consultants for NewLink Genetics Inc. and are also shareholders in this company. GCP also discloses interests in Incyte as a shareholder and in Kyn Therapeutics as a scientific advisor. ALM also discloses interests as a scientific advisor to Kyn Therapeutics. 
Figure Legends:

Figure 1. Inflammatory and immunologic processes during tumourigenesis. (a) Local immune surveillance and anti-tumour immunity prevents pre-malignant lesions from forming tumours in most people (green boxes). In some people, local inflammatory processes associated with pre-malignant lesions promote immune regulatory (tolerogenic) processes that overcome immune surveillance and establish robust immune checkpoints at clinical presentation of cancer (purple boxes). Increasing rates of cell death and consequent release of damage-associated molecular patterns (DAMPs) such as cellular DNA are common features of developing tumour lesions, which may contribute to dominance of tolerogenic processes that promote tumour development. (b) Changes in the inflammatory and immunologic status of primary tumour lesions are accompanied by parallel changes in tumour-draining lymph nodes (TDLNs) that promote development of regulatory antigen presenting cells (APCs) and Tregs, which suppress generation of stimulatory APCs and helper and effector T cells. Elevated Trp and Arg catabolism may manifest in primary tumour lesions, in TDLNs or in both. Hence, effective cancer therapy must disrupt immune checkpoints to allow anti-tumour immunity to manifest and must also prevent re-emergence of tolerogenic processes following therapy that may cause tumour relapse.

Figure 2. Inflammatory processes stimulate Trp and Arg catabolism in the TME. Inflammation increases local cell stress and death rates. DAMPs released by stressed and dying cells excite DAMP sensors (such as TLRs, NLRs and cGAS-STING) that activate immune cells directly or indirectly. Activated immune cells may acquire regulatory or stimulatory functions (purple and green coloured cells, respectively). Interferons (IFNs) have prominent roles in activating immune cells and are also released by activated innate and adaptive immune cells. IFNs and other inflammatory cytokines also stimulate expression of genes encoding enzymes that promote Trp and Arg catabolism, including IDO, ARG1 and iNOS (shown in blue). TDO is not IFN responsive but is induced by dietary stress and glucocorticoids. Sustained Trp and Arg catabolism suppresses local immune responses via mechanisms dependent on GCN2, mTOR or AHR that impact tumour growth and therapy, and may also contribute to comorbidities commonly associated with cancer.

Figure 3. Immunologic and neurologic consequences of Trp catabolism relevant to cancer. Cells expressing IDO or TDO generate downstream Trp catabolites that impact immunologic or neurologic processes. Kynurenine (Kyn), 3-hydroxykynurenine (3HK) and 3- 
hydroxyanthranilic acid (HAA) suppress immune responses, while xanthurenic acid (XA) inhibits BH4 synthesis required to generate effector T cells. Several Trp catabolites generated downstream, kynurenic acid (KA), quinolinic acid (QA) and picolinic acid (PA), are neuroactive. Trp is also the obligate substrate for synthesis of serotonin (also known as 5-hydroxytryptamine $(5 \mathrm{HT}))$ and melatonin, which promote positive neurologic behaviours. Sustained Trp catabolism may drive neurologic comorbidities commonly associated with cancer development or linked to some cancer therapies. Cells expressing iNOS or ARG1 catabolize Arg to generate nitric oxide and polyamines, which impact vascular, immunologic and other physiologic processes. Other Abbreviations: Tryptophan hydrolase (TPH), kynurenine monooxygenase (KMO), anthranilic acid (AA), kynureninase (KYNU), kynurenine aminotransferase (KAT), 3-hydroxyanthranic acid 3,4-dioxygenase (HAAO), 2-Amino-3-carboxymuconic acid-6-semialdehyde (ACMSD), quinolinate phosphoribosyl transferase (QPRT), N-methyl-D-aspartate receptor (NMDA-R), nicotinamide adenine dinucleotide (NAD). Dashed arrows indicate multiple enzyme steps. 


\section{References}

1 Bissell, M. J. \& Hines, W. C. Why don't we get more cancer? A proposed role of the microenvironment in restraining cancer progression. Nat Med 17, 320-329 (2011).

2 Vesely, M. D. \& Schreiber, R. D. Cancer immunoediting: antigens, mechanisms, and implications to cancer immunotherapy. Ann N Y Acad Sci 1284, 1-5 (2013).

3 Dvorak, H. F. Tumors: wounds that do not heal. Similarities between tumor stroma generation and wound healing. N Engl J Med 315, 1650-1659 (1986).

4 Casero, R. A., Jr., Murray Stewart, T. \& Pegg, A. E. Polyamine metabolism and cancer: treatments, challenges and opportunities. Nature reviews. Cancer, doi:10.1038/s41568018-0050-3 (2018).

5 Garber, K. A new cancer immunotherapy suffers a setback. Science 360, 588 (2018).

6 Mitchell, T. C. et al. Epacadostat Plus Pembrolizumab in Patients With Advanced Solid Tumors: Phase I Results From a Multicenter, Open-Label Phase I/II Trial (ECHO202/KEYNOTE-037). J Clin Oncol, JCO2018789602, (2018).

7 Muller, A. J., Manfredi, M., Zakharia, Y. \& Prendergast, G. C. IDO inhibitors for cancer treatment: lessons from ECHO-301. . Semin Immunopathol In press, doi:10.1007/s00281-018-0702-0 (2018).

8 Seymour, R. L., Ganapathy, V., Mellor, A. L. \& Munn, D. H. A high-affinity, tryptophanselective amino acid transport system in human macrophages. J Leukoc Biol 80, 13201327 (2006).

9 Ron, D. Translational control in the endoplasmic reticulum stress response. J Clin Invest 110, 1383-1388 (2002).

10 Lehman, S. L., Ryeom, S. \& Koumenis, C. Signaling through alternative Integrated Stress Response pathways compensates for GCN2 loss in a mouse model of soft tissue sarcoma. Sci Rep 5, 11781 (2015).

11 Mossmann, D., Park, S. \& Hall, M. N. mTOR signalling and cellular metabolism are mutual determinants in cancer. Nature reviews. Cancer 18, 744-757 (2018).

12 Cormerais, Y. et al. Genetic Disruption of the Multifunctional CD98/LAT1 Complex Demonstrates the Key Role of Essential Amino Acid Transport in the Control of mTORC1 and Tumor Growth. Cancer Res 76, 4481-4492 (2016).

13 Esaki, N. et al. ASC amino acid transporter 2, defined by enzyme-mediated activation of radical sources, enhances malignancy of GD2-positive small-cell lung cancer. Cancer Sci 109, 141-153 (2018). 
14 Wyant, G. A. et al. mTORC1 Activator SLC38A9 Is Required to Efflux Essential Amino Acids from Lysosomes and Use Protein as a Nutrient. Cell 171, 642-654 e612 (2017).

15 Muller, A. J. et al. Chronic inflammation that facilitates tumor progression creates local immune suppression by inducing indoleamine 2,3 dioxygenase. Proc Natl Acad Sci U S A 105, 17073-17078 (2008). The first genetic study of IDO establishing its key contributions to formation of a pathogenic inflammatory milieu that is critical for malignant development.

16 Smith, C. et al. IDO is a nodal pathogenic driver of lung cancer and metastasis development. Cancer Discovery 2, 722-735 (2012). This study offered genetic proofs that IDO is crucial for tumour formation, vasculogenesis, metastasis and MDSC activation and recruitment.

17 Metz, R. et al. IDO inhibits a tryptophan sufficiency signal that stimulates mTOR: A novel IDO effector pathway targeted by D-1-methyl-tryptophan. Oncoimmunology 1, 14601468 (2012).

18 Mautino, M. R. et al. Abstract 4076: A novel prodrug of indoximod with enhanced pharmacokinetic properties. Cancer Research 77, (2017).

19 Friberg, M. et al. Indoleamine 2,3-dioxygenase contributes to tumor cell evasion of T cell-mediated rejection. International Journal of Cancer 101, 151-155 (2002).

20 Muller, A. J., DuHadaway, J. B., Donover, P. S., Sutanto-Ward, E. \& Prendergast, G. C. Inhibition of indoleamine 2,3-dioxygenase, an immunoregulatory target of the cancer suppression gene Bin1, potentiates cancer chemotherapy. Nat Med 11, 312-319 (2005). First study to link IDO to a cancer pathway and to show that IDO inhibitors can exert robust anti-tumour effects if combined with DNA-damaging chemotherapy.

21 Hou, D. Y. et al. Inhibition of indoleamine 2,3-dioxygenase in dendritic cells by stereoisomers of 1-methyl-tryptophan correlates with antitumor responses. Cancer Res 67, 792-801 (2007).

22 Lemos, H. et al. STING Promotes the Growth of Tumors Characterized by Low Antigenicity via IDO Activation. Cancer Res 76, 2076-2081 (2016). This study shows that STING promotes growth of poorly immunogenic tumours by stimulating DCs in TDLNs to express IDO.

23 Weiner, G. J. CpG oligodeoxynucleotide-based therapy of lymphoid malignancies. Advanced drug delivery reviews 61, 263-267 (2009).

24 Unterholzner, L. The interferon response to intracellular DNA: why so many receptors? Immunobiology 218, 1312-1321 (2013). 
25 Prendergast, G. C., Metz, R., Muller, A. J., Merlo, L. M. \& Mandik-Nayak, L. IDO2 in Immunomodulation and Autoimmune Disease. Front Immunol 5, 585 (2014).

26 Badawy, A. A. Tryptophan availability for kynurenine pathway metabolism across the life span: Control mechanisms and focus on aging, exercise, diet and nutritional supplements. Neuropharmacology 112, 248-263 (2017).

27 Morris, G., Carvalho, A. F., Anderson, G., Galecki, P. \& Maes, M. The Many Neuroprogressive Actions of Tryptophan Catabolites (TRYCATs) that may be Associated with the Pathophysiology of Neuro-Immune Disorders. Current pharmaceutical design 22, 963-977 (2016).

28 Thomas, S. R., Mohr, D. \& Stocker, R. Nitric oxide inhibits indoleamine 2,3-dioxygenase activity in interferon-gamma primed mononuclear phagocytes. Journal of Biological Chemistry 269, 14457-14464 (1994).

29 Hesterberg, R. S., Cleveland, J. L. \& Epling-Burnette, P. K. Role of Polyamines in Immune Cell Functions. Med Sci (Basel) 6, doi:10.3390/medsci6010022 (2018).

30 Boutard, V. et al. Transforming growth factor-beta stimulates arginase activity in macrophages. Implications for the regulation of macrophage cytotoxicity. Journal of immunology 155, 2077-2084 (1995).

31 Pallotta, M. T. et al. Indoleamine 2,3-dioxygenase is a signaling protein in long-term tolerance by dendritic cells. Nat Immunol 12, 870-878 (2011).

32 Theate, I. et al. Extensive profiling of the expression of the indoleamine 2,3-dioxygenase 1 protein in normal and tumoral human tissues. Cancer immunology research 3, 161-172 (2015).

33 Munn, D. H. \& Mellor, A. L. IDO in the Tumor Microenvironment: Inflammation, CounterRegulation, and Tolerance. Trends Immunol 37, 193-207 (2016).

34 El-Zaatari, M. et al. Indoleamine 2,3-Dioxygenase 1, Increased in Human Gastric PreNeoplasia, Promotes Inflammation and Metaplasia in Mice and Is Associated With Type II Hypersensitivity/Autoimmunity. Gastroenterology 154, 140-153 e117 (2018).

35 Platten, M., Wick, W. \& Van den Eynde, B. J. Tryptophan catabolism in cancer: beyond IDO and tryptophan depletion. Cancer Res 72, 5435-5440 (2012).

36 Prendergast, G. C., Malachowski, W. P., DuHadaway, J. B. \& Muller, A. J. Discovery of IDO1 Inhibitors: From Bench to Bedside. Cancer Res 77, 6795-6811 (2017).

37 Uyttenhove, C. et al. Evidence for a tumoral immune resistance mechanism based on tryptophan degradation by indoleamine 2,3-dioxygenase. Nat Med 9, 1269 - 1274 
(2003). An early report highlighting that elevated IDO expression was a common TME feature and that IDO inhibition could enhance $T$ cell accumulation in the TME.

38 Witkiewicz, A. K. et al. Genotyping and expression analysis of IDO2 in human pancreatic cancer: a novel, active target. J Am Coll Surg 208, 781-787; discussion 787-789 (2009).

39 Brandacher, G. et al. Prognostic value of indoleamine 2,3-dioxygenase expression in colorectal cancer: effect on tumor-infiltrating T cells. Clin Cancer Res 12, 1144-1151 (2006). One of the earliest studies to establish that high IDO activity in human tumours tends to associate with a poor prognosis.

$40 \mathrm{Yu}, \mathrm{J}$. et al. Upregulated expression of indoleamine 2, 3-dioxygenase in primary breast cancer correlates with increase of infiltrated regulatory $T$ cells in situ and lymph node metastasis. Clin Dev Immunol 2011, 1-10, (2011).

41 Qian, F. et al. Efficacy of levo-1-methyl tryptophan and dextro-1-methyl tryptophan in reversing indoleamine-2,3-dioxygenase-mediated arrest of T-cell proliferation in human epithelial ovarian cancer. Cancer Res 69, 5498-5504, (2009).

42 Feder-Mengus, C. et al. High expression of indoleamine 2,3-dioxygenase gene in prostate cancer. Eur J Cancer 44, 2266-2275, doi:10.1016/j.ejca.2008.05.023 (2008).

43 Brody, J. R. et al. Expression of indoleamine 2,3-dioxygenase in metastatic malignant melanoma recruits regulatory $T$ cells to avoid immune detection and affects survival. Cell Cycle 8, 1930-1934, (2009).

44 Corm, S. et al. Indoleamine 2,3-dioxygenase activity of acute myeloid leukemia cells can be measured from patients' sera by HPLC and is inducible by IFN-gamma. Leuk Res 33, 490-494, (2009).

45 Opitz, C. A. et al. An endogenous tumour-promoting ligand of the human aryl hydrocarbon receptor. Nature 478, 197-203, (2011). The first study to link TDO activity with $A h R$ signaling and showing that this pathway promotes tumour development.

46 D'Amato, N. C. et al. A TDO2-AhR signaling axis facilitates anoikis resistance and metastasis in triple-negative breast cancer. Cancer Res 75, 4651-4664, (2015).

47 Wei, L. et al. High Indoleamine 2,3-Dioxygenase Is Correlated With Microvessel Density and Worse Prognosis in Breast Cancer. Front Immunol 9, 724, (2018).

48 Sinclair, L. V. et al. Control of amino-acid transport by antigen receptors coordinates the metabolic reprogramming essential for T cell differentiation. Nat Immunol 14, 500-508, (2013). This study reveals an obligatory requirement for activated T cells to up-regulate amino acid transporter activity to stimulate $m T o R$ and differentiate into effector $T$ cells. 
49 Lee, G. K. et al. Tryptophan deprivation sensitizes activated T cells to apoptosis prior to cell division. Immunology 107, 1-9 (2002).

50 Munn, D. H. et al. GCN2 Kinase in T Cells Mediates Proliferative Arrest and Anergy Induction in Response to Indoleamine 2,3-Dioxygenase. Immunity 22, 1-10 (2005). This study identifies a critical requirement for GCN2 signaling for T cells to proliferate and differentiate.

51 Rodriguez, P. C., Quiceno, D. G. \& Ochoa, A. C. L-arginine availability regulates Tlymphocyte cell-cycle progression. Blood 109, 1568-1573 (2007). This study links Arg catabolism to blocking T cell entry into cell cycle via a GCN2-dependent mechanism.

52 Sharma, M. D. et al. The PTEN pathway in Tregs is a critical driver of the suppressive tumor microenvironment. Sci Adv 1, e1500845, (2015).

53 Sharma, M. D. et al. Reprogrammed foxp3(+) regulatory T cells provide essential help to support cross-presentation and CD8(+) T cell priming in naive mice. Immunity 33, 942954 (2010).

54 Sharma, M. D. et al. Plasmacytoid dendritic cells from mouse tumor-draining lymph nodes directly activate mature Tregs via IDO. J Clin Invest 117, 2570-2582, (2007).

55 Sharma, M. D. et al. An inherently bi-functional subset of Foxp3+ Treg/T-helper cells is controlled by the transcription factor Eos. Immunity 38, 998-1012 (2013).

56 Munn, D. H. et al. Expression of indoleamine 2,3-dioxygenase by plasmacytoid dendritic cells in tumor-draining lymph nodes. J Clin Invest 114, 280-290 (2004). This study identifies IDO-expressing DCs in tumour-draining LNs as potent regulators of $T$ cell immunity.

57 Munn, D. H. et al. Potential regulatory function of human dendritic cells expressing IDO. Science 297, 1867-1870 (2002).

58 Chen, W., Liang, X., Peterson, A. J., Munn, D. H. \& Blazar, B. R. The indoleamine 2,3dioxygenase pathway is essential for human plasmacytoid dendritic cell-induced adaptive T regulatory cell generation. Journal of immunology 181, 5396-5404, (2008).

59 Lee, J. R. et al. Pattern of recruitment of immunoregulatory antigen-presenting cells in malignant melanoma. Lab Invest 83, 1457-1466 (2003).

60 Montero, A. J., Diaz-Montero, C. M., Kyriakopoulos, C. E., Bronte, V. \& Mandruzzato, S. Myeloid-derived suppressor cells in cancer patients: a clinical perspective. J Immunother 35, 107-115, (2012).

61 Bronte, V. \& Zanovello, P. Regulation of immune responses by L-arginine metabolism. Nat Rev Immunol 5, 641-654 (2005). 
62 Holmgaard, R. B. et al. Tumor-Expressed IDO Recruits and Activates MDSCs in a TregDependent Manner. Cell reports 13, 412-424, (2015). This report showed that IDO inhibitors could phenocopy IDO genetic blockade in blunting MDSC recruitment and activation in the TME.

63 Gielen, P. R. et al. Elevated levels of polymorphonuclear myeloid-derived suppressor cells in patients with glioblastoma highly express S100A8/9 and arginase and suppress T cell function. Neuro Oncol 18, 1253-1264, (2016).

64 Zhang, H. et al. Fibrocytes represent a novel MDSC subset circulating in patients with metastatic cancer. Blood, 1105-1113. (2013).

65 Mellor, A. L. et al. Cutting Edge: CpG Oligonucleotides Induce Splenic CD19+ Dendritic Cells to Acquire Potent IDO-Dependent T Cell Regulatory Functions via IFN Type 1 Signaling. Journal of immunology 175, 5601-5605 (2005).

66 Ravishankar, B. et al. Tolerance to apoptotic cells is regulated by indoleamine 2,3dioxygenase. Proc Natl Acad Sci U S A 109, 3909-3914, (2012).

67 Ravishankar, B. et al. The amino acid sensor GCN2 inhibits inflammatory responses to apoptotic cells promoting tolerance and suppressing systemic autoimmunity. Proc Natl Acad Sci U S A 112, 10774-10779, (2015).

68 Ravishankar, B. et al. Marginal zone CD169+ macrophages coordinate apoptotic celldriven cellular recruitment and tolerance. Proceedings of the National Academy of Sciences of the United States of America 111, 4215-4220, (2014).

69 Huang, L. et al. Cutting edge: DNA sensing via the STING adaptor in myeloid dendritic cells induces potent tolerogenic responses. Journal of immunology 191, 3509-3513, (2013).

70 Huang, L. et al. Engineering DNA Nanoparticles as Immunomodulatory Reagents that Activate Regulatory T Cells. Journal of immunology 188, 4913-4920, (2012).

71 Munn, D. H., Sharma, M. D. \& Mellor, A. L. Ligation of B7-1/B7-2 by Human CD4(+) T Cells Triggers Indoleamine 2,3-Dioxygenase Activity in Dendritic Cells. Journal of immunology 172, 4100-4110 (2004).

72 Baban, B. et al. Physiologic Control of IDO Competence in Splenic Dendritic Cells. Journal of immunology 187, 2329-2335, (2011).

73 Xia, T., Konno, H., Ahn, J. \& Barber, G. N. Deregulation of STING Signaling in Colorectal Carcinoma Constrains DNA Damage Responses and Correlates With Tumorigenesis. Cell reports 14, 282-297, (2016). This study identified correlations 
between reduced STING signaling in human colorectal carcinoma, reduced responses to DNA-damage and tumourigenesis.

74 Ahn, J., Xia, T., Rabasa Capote, A., Betancourt, D. \& Barber, G. N. Extrinsic PhagocyteDependent STING Signaling Dictates the Immunogenicity of Dying Cells. Cancer Cell 33, 862-873 (2018).

75 Shinde, R. et al. Apoptotic cell-induced AhR activity is required for immunological tolerance and suppression of systemic lupus erythematosus in mice and humans. Nat Immunol 19, 571-582, (2018).

76 Romani, L. \& Puccetti, P. Protective tolerance to fungi: the role of IL-10 and tryptophan catabolism. Trends Microbiol 14, 183-189 (2006).

77 DiNatale, B. C. et al. Kynurenic acid is a potent endogenous aryl hydrocarbon receptor ligand that synergistically induces interleukin- 6 in the presence of inflammatory signaling. Toxicological sciences : an official journal of the Society of Toxicology 115, 8997, (2010).

78 Duarte, J. H., Di Meglio, P., Hirota, K., Ahlfors, H. \& Stockinger, B. Differential influences of the aryl hydrocarbon receptor on Th17 mediated responses in vitro and in vivo. PLoS One 8, e79819, (2013).

79 Mezrich, J. D. et al. An interaction between kynurenine and the aryl hydrocarbon receptor can generate regulatory T cells. Journal of immunology 185, 3190-3198, (2010).

80 Nguyen, N. T. et al. Aryl hydrocarbon receptor negatively regulates dendritic cell immunogenicity via a kynurenine-dependent mechanism. Proc Natl Acad Sci U S A 107, 19961-19966, (2010).

81 Vogel, C. F., Goth, S. R., Dong, B., Pessah, I. N. \& Matsumura, F. Aryl hydrocarbon receptor signaling mediates expression of indoleamine 2,3-dioxygenase. Biochem Biophys Res Commun 375, 331-335, (2008).

82 Litzenburger, U. M. et al. Constitutive IDO expression in human cancer is sustained by an autocrine signaling loop involving IL-6, STAT3 and the AHR. Oncotarget 5, 10381051, (2014).

83 Feng, S., Cao, Z. \& Wang, X. Role of aryl hydrocarbon receptor in cancer. Biochim Biophys Acta 1836, 197-210, (2013).

84 Lewis, H. C., Chinnadurai, R., Bosinger, S. E. \& Galipeau, J. The IDO inhibitor 1-methyl tryptophan activates the aryl hydrocarbon receptor response in mesenchymal stromal cells. Oncotarget 8, 91914-91927, (2017). 
85 Ehrlich, A. K. \& Kerkvliet, N. I. Is chronic AhR activation by rapidly metabolized ligands safe for the treatment of immune-mediated diseases? Curr Opin Toxicol 2, 72-78, (2017).

86 Hayashi, T. et al. 3-Hydroxyanthranilic acid inhibits PDK1 activation and suppresses experimental asthma by inducing T cell apoptosis. Proc Natl Acad Sci U S A 104, 1861918624 (2007).

87 Yan, Y. et al. IDO upregulates regulatory T cells via tryptophan catabolite and suppresses encephalitogenic $T$ cell responses in experimental autoimmune encephalomyelitis. Journal of immunology 185, 5953-5961, (2010).

88 Cronin, S. J. F. et al. The metabolite BH4 controls T cell proliferation in autoimmunity and cancer. Nature 563, 564-568, (2018).

89 Adams, S. et al. Involvement of the kynurenine pathway in human glioma pathophysiology. PLoS One 9, e112945, (2014).

90 Sahm, F. et al. The endogenous tryptophan metabolite and NAD+ precursor quinolinic acid confers resistance of gliomas to oxidative stress. Cancer Res 73, 3225-3234, (2013).

91 Triplett, T. A. et al. Reversal of indoleamine 2,3-dioxygenase-mediated cancer immune suppression by systemic kynurenine depletion with a therapeutic enzyme. Nat Biotechnol, doi:10.1038/nbt.4180 (2018).

92 Sculier, J. P. et al. Medical anticancer treatment of lung cancer associated with comorbidities: a review. Lung Cancer 87, 241-248, (2015).

93 Capuron, L. \& Dantzer, R. Cytokines and depression: the need for a new paradigm. Brain, behavior, and immunity 17 Suppl 1, S119-124 (2003).

94 Sui, H. et al. 5-hydroxytryptamine receptor (5-HT1DR) promotes colorectal cancer metastasis by regulating Axin1/beta-catenin/MMP-7 signaling pathway. Oncotarget 6 , 25975-25987, (2015).

95 Gwynne, W. D. et al. Serotonergic system antagonists target breast tumor initiating cells and synergize with chemotherapy to shrink human breast tumor xenografts. Oncotarget 8, 32101-32116, (2017).

$96 \mathrm{Kim}, \mathrm{H}$. et al. Brain indoleamine 2,3-dioxygenase contributes to the comorbidity of pain and depression. J Clin Invest 122, 2940-2954, (2012).

97 Huang, L. et al. Virus Infections Incite Pain Hypersensitivity by Inducing Indoleamine 2,3 Dioxygenase. PLoS Pathog 12, e1005615, (2016). 
98 LaVoy, E. C., Fagundes, C. P. \& Dantzer, R. Exercise, inflammation, and fatigue in cancer survivors. Exerc Immunol Rev 22, 82-93 (2016).

99 Beatty, G. L. et al. First-in-Human Phase I Study of the Oral Inhibitor of Indoleamine 2,3Dioxygenase-1 Epacadostat (INCB024360) in Patients with Advanced Solid Malignancies. Clin Cancer Res 23, 3269-3276, (2017).

100 Cheong, J. E., Ekkati, A. \& Sun, L. A patent review of IDO1 inhibitors for cancer. Expert opinion on therapeutic patents 28, 317-330, (2018).

101 Fuertes, M. B. et al. Host type I IFN signals are required for antitumor CD8+ T cell responses through CD8\{alpha\}+ dendritic cells. J Exp Med 208, 2005-2016, (2011). This study shows that IFN-I signals mediated by DCs in the TME promote effector T cell responses.

102 Woo, S. R. et al. STING-Dependent Cytosolic DNA Sensing Mediates Innate Immune Recognition of Immunogenic Tumors. Immunity 41, 830-842 (2014). This and the next two references show that STING/IFN-I signaling incites immunity directed at immunogenic tumours and that synthetic STING agonists amplify this anti-tumour response.

103 Deng, L. et al. STING-dependent Cytosolic DNA Sensing Promotes Radiation-induced Type I interferon-dependent Antitumor Immunity in Immunogenic Tumors. Immunity 41, 843-852 (2014).

104 Corrales, L. et al. Direct Activation of STING in the Tumor Microenvironment Leads to Potent and Systemic Tumor Regression and Immunity. Cell reports 11, 1018-1030, (2015).

$105 \mathrm{Li}, \mathrm{T}$. et al. Antitumor Activity of cGAMP via Stimulation of cGAS-cGAMP-STING-IRF3 Mediated Innate Immune Response. Sci Rep 6, 19049, (2016).

106 Lemos, H. et al. Activation of the STING Adaptor Attenuates Experimental Autoimmune Encephalitis. Journal of immunology 192, 5571-5578 (2014).

107 Aya, F. et al. Life-threatening colitis and complete response with ipilimumab in a patient with metastatic BRAF-mutant melanoma and rheumatoid arthritis. ESMO Open 1, e000032, doi:10.1136/esmoopen-2015-000032 (2016).

108 De Martin, E. et al. Characterization of liver injury induced by cancer immunotherapy using immune checkpoint inhibitors. J Hepatol 68, 1181-1190, (2018).

109 Menzies, A. M. et al. Anti-PD-1 therapy in patients with advanced melanoma and preexisting autoimmune disorders or major toxicity with ipilimumab. Ann Oncol 28, 368376, (2017). 
110 Johnson, D. B. et al. Ipilimumab Therapy in Patients With Advanced Melanoma and Preexisting Autoimmune Disorders. JAMA Oncol 2, 234-240, (2016).

111 Banerjee, T. et al. A key in vivo antitumor mechanism of action of natural product-based brassinins is inhibition of indoleamine 2,3-dioxygenase. Oncogene 27, 2851-2857, (2008).

112 Ursu, R. et al. Intracerebral injection of $\mathrm{CpG}$ oligonucleotide for patients with de novo glioblastoma-A phase II multicentric, randomised study. Eur J Cancer 73, 30-37, (2017).

113 Moreno Ayala, M. A. et al. Dual activation of Toll-like receptors 7 and 9 impairs the efficacy of antitumor vaccines in murine models of metastatic breast cancer. $J$ Cancer Res Clin Oncol 143, 1713-1732, (2017).

114 Tarhini, A. A., Gogas, H. \& Kirkwood, J. M. IFN-alpha in the treatment of melanoma. Journal of immunology 189, 3789-3793, (2012).

115 Mojic, M., Takeda, K. \& Hayakawa, Y. The Dark Side of IFN-gamma: Its Role in Promoting Cancer Immunoevasion. Int J Mol Sci 19, doi:10.3390/ijms19010089 (2017).

116 McMasters, K. M. et al. Final Results of the Sunbelt Melanoma Trial: A Multi-Institutional Prospective Randomized Phase III Study Evaluating the Role of Adjuvant High-Dose Interferon Alfa-2b and Completion Lymph Node Dissection for Patients Staged by Sentinel Lymph Node Biopsy. J Clin Oncol 34, 1079-1086, (2016).

117 Mautino, M. R. et al. in 104th Annual Meeting of the American Association for Cancer Research Abstract 491 (2013).

118 Nayak, A. et al. in European Society for Medical Oncology Annual Meeting (2015).

119 Siu, L. L. et al. in American Association for Cancer Research Annual Meeting Abstract CT116 (2017).

120 Reardon, D. et al. in Society of Neuro-Oncology Annual Meeting Abstract ATIM-29 (2017).

121 Sahebjam, S. et al. in Society for Immunotherapy of Cancer Annual Meeting Abstract P148 (2017).

122 Mautino, M. et al. in American Association for Cancer Research Annual Meeting Abstract 4076 (2017).

123 Herbst, R. S. et al. Predictive correlates of response to the anti-PD-L1 antibody MPDL3280A in cancer patients. Nature 515, 563-567, (2014).

124 Spranger, S. et al. Up-regulation of PD-L1, IDO, and T(regs) in the melanoma tumor microenvironment is driven by CD8(+) T cells. Science translational medicine $\mathbf{5}$, 200ra116, doi:10.1126/scitransImed.3006504 (2013). 
125 Holmgaard, R. B., Zamarin, D., Munn, D. H., Wolchok, J. D. \& Allison, J. P. Indoleamine 2,3-dioxygenase is a critical resistance mechanism in antitumor T cell immunotherapy targeting CTLA-4. J Exp Med 210, 1389-1402, (2013). First study to suggest that IDO blockade could empower immune checkpoint therapy.

126 Spranger, S. et al. Mechanism of tumor rejection with doublets of CTLA-4, PD-1/PD-L1, or IDO blockade involves restored IL-2 production and proliferation of CD8(+) T cells directly within the tumor microenvironment. Journal for Immunotherapy of Cancer 2, 3, (2014).

127 Wainwright, D. A. et al. Durable therapeutic efficacy utilizing combinatorial blockade against IDO, CTLA-4, and PD-L1 in mice with brain tumors. Clinical Cancer Research 20, 5290-5301, (2014).

128 Zakharia, Y. et al. in American Association for Cancer Research Annual Meeting. Abstract CT117.

129 Zakharia, Y. et al. in ASCO Annual Meeting. Abstract 3075.

130 Zakharia, Y., Munn, D., Link, C., Vahanian, N. \& Kennedy, E. in Society of NeuroOncology Annual Meeting. vi13-vi14, Abstract ACTR-53.

131 Smith, D. C. et al. Epacadostat plus pembrolizumab in patients with advanced urothelial carcinoma: Preliminary phase I/II results of ECHO-202/KEYNOTE-037. Journal of Clinical Oncology 35, 4503-4503, (2017).

132 Lara, P. et al. Epacadostat plus pembrolizumab in patients with advanced RCC: Preliminary phase I/II results from ECHO-202/KEYNOTE-037. Journal of Clinical Oncology 35, 4515-4515, (2017).

133 Gangadhar, T. C. et al. Efficacy and safety of epacadostat plus pembrolizumab treatment of NSCLC: Preliminary phase I/II results of ECHO-202/KEYNOTE-037. Journal of Clinical Oncology 35, 9014-9014, (2017).

134 Hamid, O. et al. Epacadostat plus pembrolizumab in patients with SCCHN: Preliminary phase I/II results from ECHO-202/KEYNOTE-037. Journal of Clinical Oncology 35, 6010-6010, (2017).

135 Genentech. A Study of GDC-0919 and Atezolizumab Combination Treatment in Participants With Locally Advanced or Metastatic Solid Tumors (NCT02471846). Clinical Trial (2018).

136 Long, G. V. et al. Epacadostat $(E)$ plus pembrolizumab $(P)$ versus pembrolizumab alone in patients (pts) with unresectable or metastatic melanoma: Results of the phase 3 ECHO-301/KEYNOTE-252 study. Clinical Oncology 36 (2018). 
137 Galluzzi, L., Buque, A., Kepp, O., Zitvogel, L. \& Kroemer, G. Immunological Effects of Conventional Chemotherapy and Targeted Anticancer Agents. Cancer Cell 28, 690-714, (2015).

$138 \mathrm{Li}, \mathrm{M}$. et al. The indoleamine 2,3-dioxygenase pathway controls complement-dependent enhancement of chemo-radiation therapy against murine glioblastoma. J Immunother Cancer 2, 21, (2014).

139 Johnson, T. S. \& Munn, D. H. Host indoleamine 2,3-dioxygenase: contribution to systemic acquired tumor tolerance. Immunological Investigations 41, 765-797, (2012).

140 Soliman, H. H. et al. A first in man phase I trial of the oral immunomodulator, indoximod, combined with docetaxel in patients with metastatic solid tumors. Oncotarget 5, 81368146, (2014).

141 Bahary, N. et al. Phase 2 trial of the indoleamine 2,3-dioxygenase pathway (IDO) inhibitor indoximod plus gemcitabine/nab-paclitaxel for the treatment of metastatic pancreas cancer: Interim analysis. ASCO Annual Meeting Proceedings, (Abstract 3020) (2016).

142 Bahary, N. et al. in ASCO Annual Meeting Proceedings. 452.

143 Emadi, A. et al. in 22nd European Hematologic Association (EHA) Congress Abstract number E-912 (Madrid, Spain, 2017).

144 Johnson, T. S. et al. in Society of Neuro-Oncology Annual Meeting Abstract PDCT-06 (2017).

145 Johnson, T. S. et al. in American Society of Pediatric Hematology Oncology Annual Meeting Abstract 4027 (2017).

146 Lugade, A. A. et al. Radiation-induced IFN-gamma production within the tumor microenvironment influences antitumor immunity. Journal of immunology 180, 31323139 (2008).

147 Ladomersky, E. et al. IDO1 Inhibition Synergizes with Radiation and PD-1 Blockade to Durably Increase Survival Against Advanced Glioblastoma. Clin Cancer Res, doi:10.1158/1078-0432.CCR-17-3573 (2018).

148 Hiniker, S. M., Chen, D. S. \& Knox, S. J. Abscopal effect in a patient with melanoma. $N$ Engl J Med 366, 2035; author reply 2035-2036, (2012).

149 Hiniker, S. M. et al. A systemic complete response of metastatic melanoma to local radiation and immunotherapy. Transl Oncol 5, 404-407 (2012).

150 Postow, M. A. et al. Immunologic correlates of the abscopal effect in a patient with melanoma. N Engl J Med 366, 925-931, (2012). 
151 Twyman-Saint Victor, C. et al. Radiation and dual checkpoint blockade activate nonredundant immune mechanisms in cancer. Nature 520, 373-377, (2015).

152 Wang, W. et al. IDO Immune Status after Chemoradiation May Predict Survival in Lung Cancer Patients. Cancer Res 78, 809-816, (2018). This study reveals strong correlations between high systemic IDO activity in lung cancer (NSCLC) patients and poor survival prospects after radio-chemotherapy. 
[AU: I note that a nearly identical table was published by you in a Cancer Research 2017 article. Although it is your table, and I am suggesting reorganization, because it was modified from a previously published version, we must obtain copyright permission to modify this. Please ensure you fill out the third party rights table mentioned in my letter so that our editorial assistant can obtain this permission. If any other figures have been modified from previously published versions, please also include them in the table and state this in the figure legend/table footnote.]

[AU: I reformatted the table to improve readability and so that it conforms to our style. Headings for the rows in the first column must be on the top of the table, and there should be a heading added for the company, rather than this being the first row. I put the drug name column first, and ordered the rows according to development stage (phase 3 on the top, preclinical on the bottom). Please check carefully for accuracy.]

Table 1. IDO1 inhibitors under preclinical and clinical evaluation. Evaluation of multiple ID01 inhibitors at a clinical stage of development has been delayed due to negative results obtained in the Phase 3 trial KEYNOTE-252/ECHO-301 (NCT02752074), a combination of the field's lead compound epacadostat with the anti-PD1 immune checkpoint therapy pembrolizumab widely expected to be efficacious ${ }^{108}$. [AU: As tables cannot include legends please move this information to the main text, You can add a footnote stating development has been delayed/terminated. Also, should ref 112 be cited instead of 108?]

\begin{tabular}{|c|c|c|c|c|c|c|c|c|}
\hline Drug name(s) & Structure & $\begin{array}{c}\text { Mechanis } \\
\text { m }\end{array}$ & $\begin{array}{l}\text { Inhibitory } \\
\text { Kinetics }\end{array}$ & $\begin{array}{l}\text { Cell-Based } \\
\text { Potency [Au: } \\
\text { Please clarify } \\
\text { what these } \\
\text { numbers are - } \\
\text { IC50 or } \\
\text { something } \\
\text { else?] }\end{array}$ & $\begin{array}{l}\text { IDO:TDO } \\
\text { Selectivity }\end{array}$ & $\begin{array}{c}\text { Company } \\
\text { [Au: Please } \\
\text { clarify what } \\
\text { the } 2^{\text {nd }} \text { names } \\
\text { are next to } \\
\text { some } \\
\text { companies.] }\end{array}$ & $\begin{array}{l}\text { Clinical } \\
\text { Entrance }\end{array}$ & $\begin{array}{l}\mathbf{P} \\
\mathbf{S} \\
{[} \\
\mathbf{p} \\
\mathbf{p} \\
\mathbf{r} \\
\mathbf{s} \\
\mathbf{a}\end{array}$ \\
\hline $\begin{array}{l}\text { D-1MT (also known as } \\
\text { Indoximod and NLG- } \\
\text { 8186) [Au:OK?] }\end{array}$ & & $\begin{array}{c}\text { Stimulates } \\
\text { mTORC1 } \\
\text { downstream } \\
\text { of } \\
\text { IDO1/TDO }\end{array}$ & NA & NA & Non-selective & NewLink & 2008 & \\
\hline $\begin{array}{l}\text { Epacadostat } \\
\text { (also known as } \\
\text { INCB024360) }\end{array}$ & & $\begin{array}{l}\text { Catalytic } \\
\text { inhibitor }\end{array}$ & $\begin{array}{l}\text { Tryptophan } \\
\text { competitive }\end{array}$ & $12 \mathrm{nM}$ & $>100$-fold & Incyte & 2012 & \\
\hline
\end{tabular}




\begin{tabular}{|c|c|c|c|c|c|c|c|c|}
\hline $\begin{array}{c}\text { BMS-986205 } \\
\text { (also known as F001287) }\end{array}$ & & $\begin{array}{c}\text { Apoptosis } \\
\text { [Au:OK?] } \\
\text { inhibitor }\end{array}$ & Irreversible & $2 \mathrm{nM}$ & $>100$-fold & $\begin{array}{l}\text { BMS } \\
\text { Flexus }\end{array}$ & 2015 & \\
\hline $\begin{array}{c}\text { Navoximod } \\
\text { (also known as NLG-919) }\end{array}$ & & $\begin{array}{l}\text { Catalytic } \\
\text { inhibitor }\end{array}$ & $\begin{array}{c}\text { Tryptophan } \\
\text { non-competitive }\end{array}$ & $75 \mathrm{nM}$ & $10-20$ fold & NewLink & 2015 & $\bar{P}$ \\
\hline PF-06840003 & & $\begin{array}{l}\text { Catalytic } \\
\text { inhibitor }\end{array}$ & $\begin{array}{c}\text { Tryptophan } \\
\text { non-competitive }\end{array}$ & $1100 \mathrm{nM}$ & $>100$-fold & $\begin{array}{l}\text { Pfizer } \\
\text { iTeos }\end{array}$ & 2016 & \\
\hline IOM2983 & $\begin{array}{c}\text { Unknown } \\
\text { Patent Published [Au: If } \\
\text { possible, please cite the } \\
\text { patent for this in the } \\
\text { reference list.] }\end{array}$ & Unknown & Unknown & Unknown & $>100$-fold & $\begin{array}{l}\text { Merck } \\
\text { IO-Met }\end{array}$ & NA & $\overline{\mathrm{P}}$ \\
\hline RG-70099 & $\begin{array}{l}\text { Unknown } \\
\text { Patent Published[Au: If } \\
\text { possible, please cite the } \\
\text { patent for this in the } \\
\text { reference list.] }\end{array}$ & Unknown & Unknown & Unknown & $\sim 5$-fold & $\begin{array}{l}\text { Roche } \\
\text { Curadev }\end{array}$ & NA & $\bar{P}$ \\
\hline
\end{tabular}

*Development in combination with pembrolizumab for melanoma terminated due to negative results (108). [AU: Should ref 108 instead be ref 112? (ALM: Note that these reference numbers need updating due to the inclusion of new citations in the revised article)]

${ }^{\dagger}$ Phase 3 trial delayed following negative results obtained from KEYNOTE-252/ECHO-301 trial of Epacadostat and pembrolizumab. [AU:OK?]

Table of Contents Summary [AU: This will appear next to the title of your article online and in the table of contents. I have written it based on your abstract; feel free to edit but please do not exceed the maximum length of 40 words.]

This Review discusses how increased catabolism of the amino acids tryptophan and arginine in the tumour microenvironment contributes to inflammatory processes that promote tumourigenesis, impede immune responses to therapy and might promote neurologic comorbidities associated with cancer. 